\title{
Comparative Studies on the Utilization of Motor oil of different varieties in Adamawa State. Usman Ezekiel Jabba ${ }^{1}$ Mbudai Dzugu Yohanna ${ }^{2}$ arniliyus Gideon Fwah ${ }^{3}$ Danladi
}

\author{
Ismaila $^{4}$
}

\author{
College of Engineering, Adamawa State Polytechnic Yola PMB 2146, Adamawa State, Nigeria
}

\begin{abstract}
Motor or Engine Oil is said to be the food for every engine. but this is only true when used within the even specifications. It is in view of this that this study compares the use of various motor oils available in the market in Adamawa State. The study revealed that $313 \%$ of the respondents use total Oil, 21.6\% use Mobile, $19.6 \%$ use AZ, 9.8\% use AP Oil, 5.9\% use Jezco Oil and others and $2.0 \%$ use Amasco respectively. And on the duration they take before changing or servicing the car, about $45.1 \%$ of respondents who responded changed Motor Oil between 25-30 days in their cars, $19.6 \%$ of the respondents used or changed Motor Oil between 30-45 days in their cars, those that use it between 20-25 days were 17.6\%, between 5.9\% of the respondents used or changed Motor Oil for between 45-55 days in their cars and other respondents used making up to 11.8\% used or changed Motor Oil in unspecified days.

Keywords: Motor oil, car, servicing, duration.
\end{abstract}

\subsection{Introduction}

The use of motor oil to lubricate is common and useful for the engine life. Vegetable oils were used in early times for lubrication, heating and lighting Mankind has been using mineral oil for thousands of years, but only in the last two hundred has it been so widely exploited.

Engine oil is slippery, it gets dirty; it drips on the garage floor, it often appears out of the exhaust pipe as black smoke and it is not welcome in the kitchen Even non-drivers know that its presence has to be checked now and again, with dire consequences if it is forgotten. Oil is thought of as the life blood of the engine. We certainly know that without it, our engine becomes a. heavy lump of scrap iron (Scott, 2005). Scott added that early cars naturally used the slippery by-products of crude oil from which their fuel was obtained to protect the sliding and rotating metal parts of the early internal combustion engines. Adjacent moving metal parts require an oil film between them to prevent seizure and as speed increases, a medium to carry away heat.

Motor oil or engine oil is used for lubrication of various internal combustion engines. The main function is to lubricate moving parts; it also cleans, inhibits corrosion, improves sealing, and cools the engine by carrying heat away from moving parts

Motor oils are derived from petroleum-based and non-petroleum-synthesized chemical compounds. Motor oils today are mainly blended bv using base oils composed of hvdrocarbons polyalphaolefins (PAO), and polyinternal olefins (PIO), thus organic compounds consisting

entirely of carbon and hydrogen. The base oils of some high-performance motor oils contain up to 20 wt- $\%$ of esters. Today we have several brands of motor oil in the market and because engine oil is significant for our vehicles, this research seeks to find out how frequent motorist utilize different varieties of the engine oil available in the market and how good such oils are to the vehicles. 
The objectives of this research is to explore more advantages and uses of Motor Oil or Engine Oil to vehicles and find out the level of utilization, varieties and most used engine oil by Motorists in Adamawa State.

When these objectives are achieved, it will help government, consumers, private business owners and the public know which Motor Oil or Engine Oil is the best for lubricating cars.

This study when completed will provides more information and materials that will contribute to academics and research works in the future, it will assist Motorists to know which of the brands of Oils is most useful for vehicles. The project will also help government and the public decide which Oil is good for consumers and insist on such a brand for usage and to encourage companies that are producing these oils to improvide on the quality of the oils they produce.

\subsection{Methodology}

\subsection{Population of the Study}

The population for the study is 51, comprises of civil servants, public servants, the self employed and others. Also, these basic attributes of the population were considered, e.g age, sex. occupation and qualification.

\subsection{Sample of the Study}

Since the population of the study is 51 , the sample of the study was taken to be 51 . The study of the entire population is necessary looking at the number.

\subsection{Sampling Techniques}

The stratified random sampling gave fair and adequate representation of all relevant strata or sub-group. This however guaranteed the accuracy of the analysis and authenticity of the sampled data.

\subsection{Methods Data Collection}

The study used both primary and secondary data. For the primary source the questionnaire was administered face-toface under direct supervision to ensure confidentiality with which response was made as well as clarifying doubts/questions arising from respondents. The administration was personally done by the researcher and the assistance of others.

\subsection{Instruments of Data Collection}

A questionnaire was developed and administered for data collection on this project. The questionnaire consists of two sections of general information and brand or varieties of motor oil/engine oil used by motorists in Adamawa State.

\subsection{Methods of Data Analysis}

To ensure easy analysis, simple percentages rating was used to draw conclusions and compares respondents' responses. Conclusions were also base on the overall findings of the research. 


\subsection{Result}

Table 1. Shows Respondents by Type of Motor Oil Used.

\begin{tabular}{|l|l|l|l|}
\hline S/NO & MOTOR OIL & RESPONDENTS & PERCENTAGE(\%) \\
\hline 1 & Jezco Oil & 3 & 5.9 \\
2 & Total Oil & 16 & 31.3 \\
3 & Mobil & 11 & 21.6 \\
4 & Ap Oil & 5 & 9.8 \\
5 & Oando Oil & 2 & 3.9 \\
6 & AZ & 10 & 19.6 \\
7 & Amasco & 1 & 2.0 \\
8 & Others & 3 & 5.9 \\
& & & \\
\hline
\end{tabular}

Table 2. Shows Respondents on the duration of use before changing Oil.

\begin{tabular}{|l|l|l|l|}
\hline S/NO & DURATION & RESPONDENTS & PERCENTAGE(\%) \\
\hline 1 & $20-25$ days & 9 & 17.6 \\
2 & $25-30$ days & 23 & 45.1 \\
3 & $30-45$ days & 10 & 19.6 \\
4 & $45-55$ days & 3 & 5.9 \\
5 & Others & 6 & 11.8 \\
& & & \\
\hline \multicolumn{2}{|l|}{ TOTAL } & 51 & 100 \\
\hline
\end{tabular}

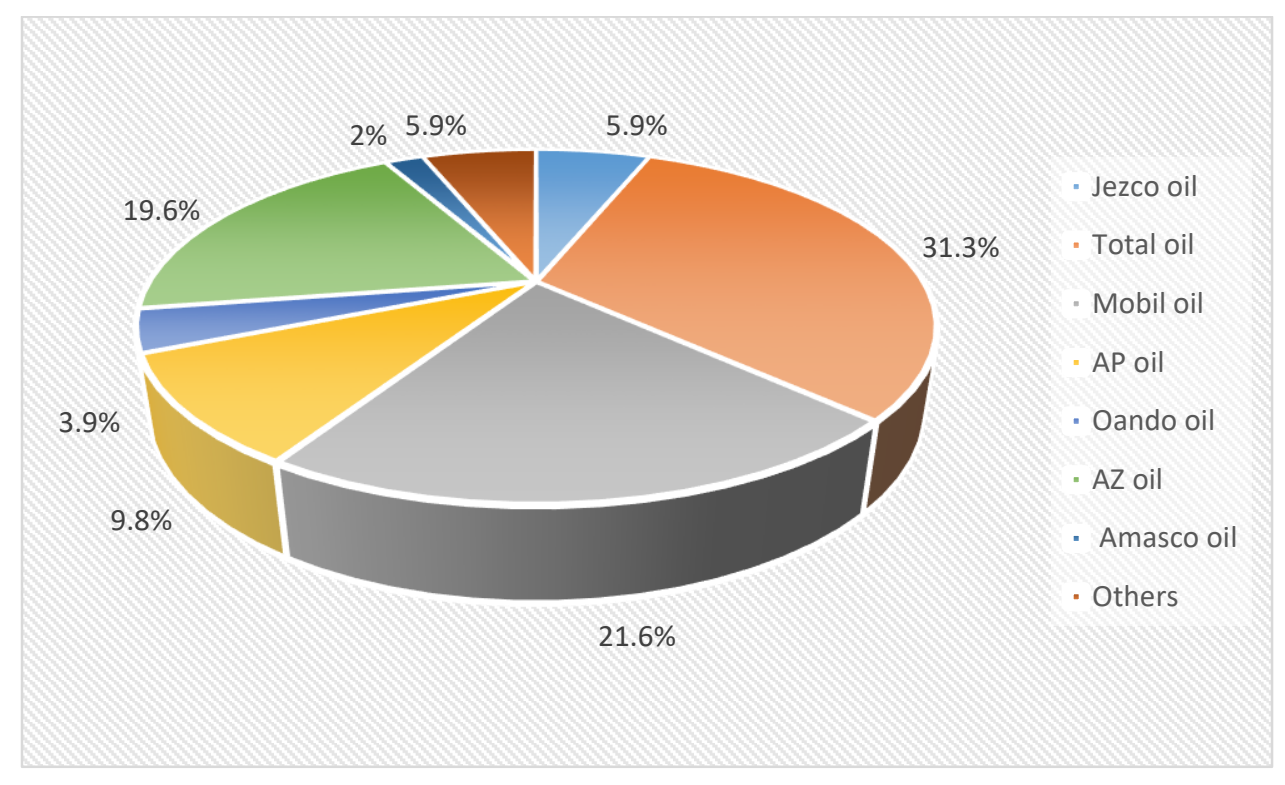

Figure 1. Types of motor oil used against percentage respondents 


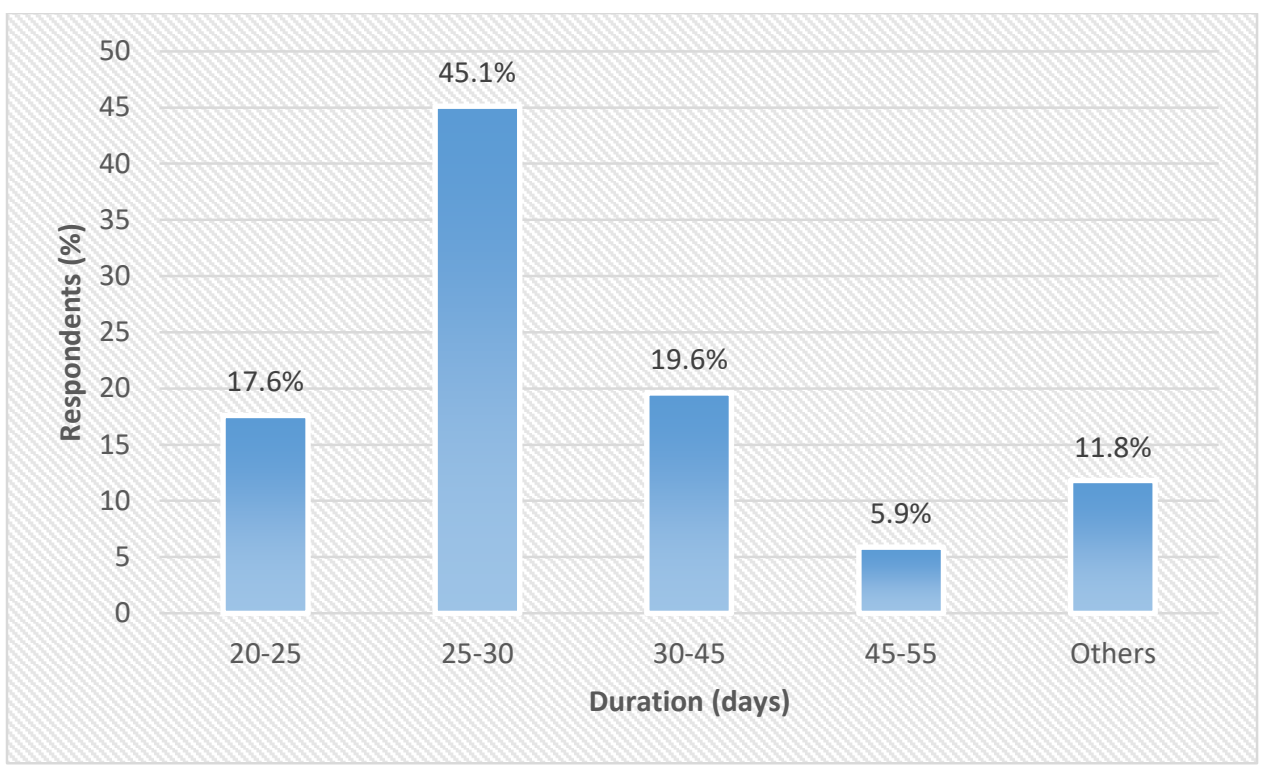

Figure 2. Percentage respondents against duration of motor oil used.

\subsection{Discussion of Results}

A total of fifty-one (51) motorists were interviewed in collecting primary data.

Figure 1. shows that, $31.3 \%$ of motorists use total Oil which represent the highest number of the respondents, and only $2 \%$ of the respondent uses Amasco oil which represent the lowest number of respondents.5.9\%,21.6\%,9.8\%,3.9\%, and $19.6 \%$ of the respondents uses Jezco oil, Mobil oil, AP oil, Oando oil and AZ oil respectively. 5.9\% of the respondents uses other types of motor oil.

Figure 2. shows $45.1 \%$ of respondents changed their Motor Oil between 25-30 days which is the majority while 5.9\% say they used or changed their Motor Oil between 45-55 days which actually is abnormal.

\subsection{Conclusion}

Majority of the respondents keeps the right duration of servicing or change of Oil in their cars, hut more awareness is needed in this regard.

From the result obtained, most motorists choose to use total Oil in their vehicles than any other type of motor oil in Adamawa State.

\subsection{Reference}

Collins C. (2007): implementing phytoremediation of petroleum hydrocarbons, Methods in Biotechnology 23:99108. Humana Press.

Corsico G., Mattei L., Roselli A., and Gommellini C. (1999): Poly(internal olefins)- Synthetic Lubricants and high performance functional fluids. Pp 53-62.

Dieter K. (1984): Lubricants and Related Products, Verlag Chemie.

Mark Lawrence M. (2011): All about Oil. Email me, mark@calsci.com.

Longhurst C. (2011): Motor Oil, Wikipedia the free encyclopedia. 
Scott G. (2005): The History of Engine Oil, Delta Oil Ltd 